\title{
Identity through Social Wearables: Designing with Finnish University Students
}

\author{
Felix Anand Epp \\ Aalto University \\ Espoo, Finland \\ felix.epp@aalto.fi \\ Andrés Lucero \\ Aalto University \\ Espoo, Finland \\ lucero@acm.com
}

\author{
Ilyena Hirskyj-Douglas \\ Aalto University \\ Espoo, Finland \\ ilyena.hirskyj-douglas@aalto.fi \\ Tapio Takala \\ Aalto University \\ Espoo, Finland \\ tapio.takala@aalto.fi
}

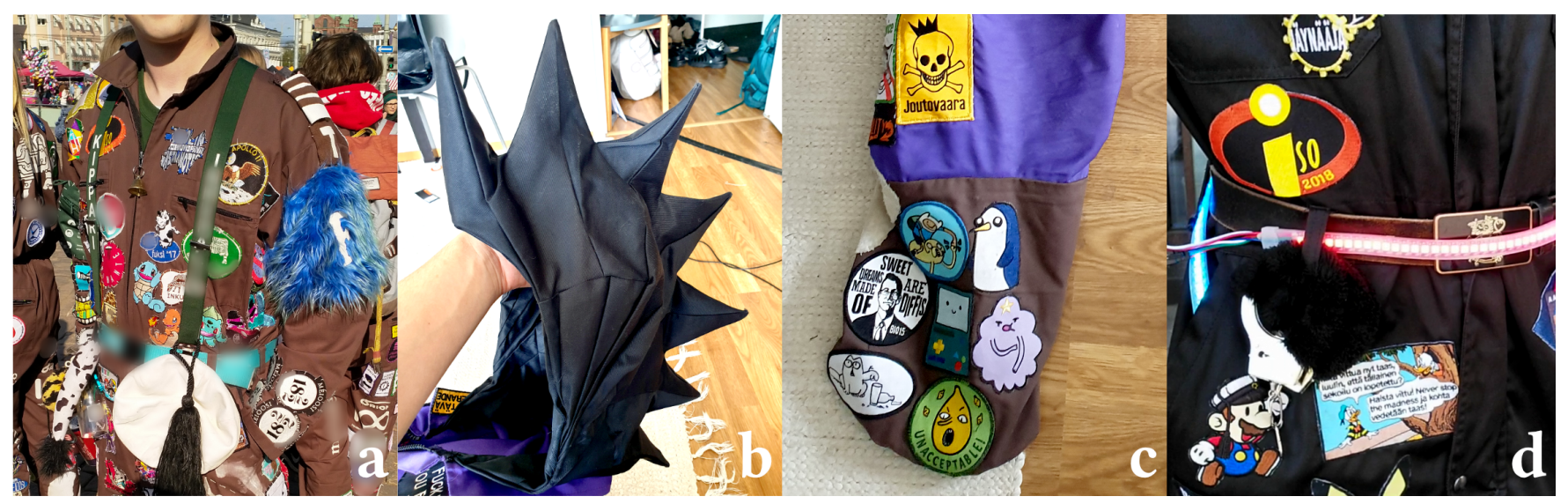

Figure 1: Modifications of student boiler suits: (a) a freshman captain identifies with a white " $F$ " on a blue felt patch, (b) a self-made hood design, (c) a different coloured pant-leg showing a relationship status, (d) a self-made LED belt.

\begin{abstract}
Wearable devices are often expressive and part of our social interactions. Fashion and ethnographic studies have argued for a perspective on socio-cultural practices in wearable design, while recent work in HCI only began to formalise social requirements for wearable design. Our work aimed to understand how cultural practices can inform wearable design. We conducted a ten-month field study on the practice of customising and wearing boiler suits (fi: opiskeljhaalarit) in the Finnish university student culture and co-designed concepts for social wearables using the dialogue-labs method. The most popular designs supported self-expression and belonging to a group, which comply with the students' cultural practices of constructing social identity. We conclude that social wearables need to let users negotiate between differentiation and belonging to become part of everyday cultural practices.
\end{abstract}

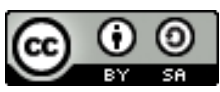

This work is licensed under a Creative Commons Attribution-ShareAlike International 4.0 License.

NordiCHI '20, October 25-29, 2020, Tallinn, Estonia

(c) 2020 Copyright held by the owner/author(s).

ACM ISBN 978-1-4503-7579-5/20/10.

https://doi.org/10.1145/3419249.3420137

\section{CCS CONCEPTS}

- Human-centered computing $\rightarrow$ Participatory design; Empirical studies in collaborative and social computing; Ethnographic studies; Empirical studies in HCI; Field studies; Empirical studies in ubiquitous and mobile computing.

\section{KEYWORDS}

Social Wearables, Co-Design, Fashion/Clothing, Cultural Practices, Ethnographic Methods

\section{ACM Reference Format:}

Felix Anand Epp, Ilyena Hirskyj-Douglas, Andrés Lucero, and Tapio Takala. 2020. Identity through Social Wearables: Designing with Finnish University Students. In Proceedings of the 11th Nordic Conference on Human-Computer Interaction: Shaping Experiences, Shaping Society (NordiCHI '20), October 25-29, 2020, Tallinn, Estonia. ACM, New York, NY, USA, 11 pages. https: //doi.org/10.1145/3419249.3420137

\section{INTRODUCTION}

With the prevalence of devices such as the smartwatch, wearable devices have started to become part of everyday life [32]. These devices can influence how bystanders perceive the wearer, which 
in turn can be used to trigger interactions between collocated people [38]. In human-computer interaction, the potential of wearables to augment collocated social interactions has received considerable attention $[9,22,36]$. These social wearables $[9,30]$ exist in the form of smart textiles, digital accessories and physical devices for gaming or collaborative work $[6,9,10,20,31,37,38]$. Recent works have implicitly contributed to this field by adding interactivity and digital expression into accessories, textiles or cosmetics [11, 23, 48].

From existing social wearables Dagan et al. [9] identified potential for future designs in "augmenting existing social signalling" and "intervening in the social situation proactively". Designing for those potentials faces challenges when wearables become part of our social interactions. For example, the "fast pace" of digital displays seems to break with the appeal of everyday clothing [10] and people are concerned about the control over the content of personal wearable displays $[11,29]$. Hence, the question of social context has to be addressed in designing social wearables [9]. The social function of technology often breaks with the function of clothes as a form of communicating self to others: self-expression to identify with a particular social group $[4,12]$. Consequently, such social wearable designs need to integrate with the social functions of dress [45].

Research has started investigating digital expression in wearables in the context of co-design of live-action role-playing $[8,9,30]$ and an auto-ethnographic study [29], which showed design can be informed by investigating the social factors of dress. Fashion and design studies [13, 14, 29] and HCI and CSCW scholars [24, 25, 53] alike argue for a perspective into socio-cultural practices. In fashion studies, a focus on practices of dress helps to gain a deeper understanding of how people build identity through clothing [13] In HCI, a practice perspective offers an understanding of the context of interaction, the role of the body (embodiment) and how people perform self (i.e. to present themselves to others) [24]. Further investigations into social context and culture are necessary to increase our understanding of how people might use wearables for various means of social practices, particularly practices of dress.

Following a practices-oriented approach [25, 53], this work uses ethnographic field studies and participatory methods in a specific culture: Finnish engineering students and their practice of wearing student caps and personalised boiler suit uniforms (as seen in figure 1). We report from a ten-month ethnographic field study and three co-design workshops using the dialogue-labs method [28] to answer the following research questions:

RQ1: How do Finnish university students customise their cultural dress, in particular boiler suits?

RQ2: How would Finnish university students use social wearables to enhance their outfits?

In this article, we first describe the background of designing social wearables and our case of Finnish student culture. Second, we report our ethnographic fieldwork findings. Third, we explain how we applied the dialogue labs method to generate concepts of social wearables with the students. Lastly, in our discussion, we point out that wearables offer versatility to dynamically mediate between standing out as an individual and conforming to a social group.

\section{BACKGROUND}

\subsection{Designing Social Wearables}

Social wearables have been defined as wearable technology that augments co-located social interaction [30]. Such technologies have already been developed from early experiments with digital badges [15] to displays integrated into clothes [10,11] and accessories [7] to augmented reality fashion [17, 29, 40]. Also kinetics [23] and interactive cosmetics [48] have extended the forms of expression with wearables.

Although the design of wearables has reached a certain maturity through the last decade, design guidelines [18] still focus on wearable technology as a single-user device or adornment. Recently Dagan et al. [9] have analysed two spaces of value for novel collocated social interactions with wearables. Firstly, by "augmenting existing social signaling", social wearables can be used to stand out [37] or enhance non-verbal communication [31, 46]. Secondly, by "intervening in the social situation proactively" wearables can enhance cooperative gaming [9,21], social matching [6] and group discussion [9].

However, such social wearables bring challenges for design by incorporating the social functions of technology with the social functions of dress $[9,45]$, namely social acceptability and selfdetermination. Social acceptability can be infringed by various factors. Firstly, visual aesthetics depend on their social interpretation [12]. For example, the aesthetics of screens with their fastchanging imagery can be perceived unfit for clothing [10]. Further, with clothing people visually express their identity [12, 29]. For example, a design of wearables that is perceived as masculine or feminine might contradict someone's gender identity [9]. Lastly, interacting with the body in novel ways creates conflicts if perceived as improper gestures $[12,54]$. Self-determination is important for people who may want to protect their privacy and control over their self-presentation. Privacy might be breached by automated sharing technology in face-to-face situations [34]. Beyond that, people fear losing control of how they present themselves to others. People manage their impression on others through language, appearance and gestures [19]. With wearables becoming active parts in expressing self, concerns on how to control such representations arise [11, 29].

In summary the existing works on designing wearables, in particular for social interaction, point towards questions of how to integrate new technical functions into dress to make them socially acceptable and fashionable. However, fashion and technology follow adoption cycles, and social norms are not static but change over time and differ depending on their social context [12, 16, 29]. Therefore more research is needed to understand better how to design for specific cultural contexts and their dynamics.

\subsection{Cultural Practices as a Starting Point}

Due to the symbolic social function of dress, wearables "[need] to offer clear symbolic value beyond functionality" [43, 45]. Tamminen \& Holmgren therefore offer [43] three cultural design spaces for future design: wearables "as technologies of discipline and control", for "mediating love, imagination and belonging" and "as autobiographical objects" [43]. From their ethnographic research on how wearables matter in people's everyday life they conclude that 
"designers of wearable technology need [to] take the symbolic world they want to be a part of as a starting point, rather than taking inspiration from the functional (or technological) cues” [43]

Fashion and clothing studies have used the perspective of dress as situated bodily practice [13] and, based on anthropology and ethnography, have investigated the practice of dress as a subjective being in the world [14, 33, 41]. Similarly, HCI and Interaction Design formulated practice-oriented design approaches [24, 25, 53]. These approaches put the subjective perspective of practices at the centre of the investigation. Understanding the social context through ethnography and participatory methods is fundamental to this methodology $[25,53]$.

In the area of social wearables and dynamic fabrics, research has started using co-design $[9,30]$ and auto-ethnography [29] to study wearables in context. Mackey and colleagues [29] argue for ongoing research to "include explorations in genuine social contexts".

\subsection{Clothing Practices in Finnish University Student Culture}

A social group has specific knowledge and generates opportunities for its members [5]. People dress themselves to stand out as individuals and at the same time fit in with a social group [13]. One social group that engages in particular practices of dress is university students. Various student cultures have practices of uniform dress codes to address roles and affiliation. The example of American college letter jackets [52] shows how such practices can pass over into contemporary fashion. These letter jackets historically are status symbols for high-performing sports students. Interestingly, they have become everyday fashion items.

In German-speaking countries, France, Belgium and the Nordic countries, students have been wearing different forms of caps. In the Nordic countries, most students receive such a cap after high school graduation. In Finland, the student cap is traditionally worn not just in faculty ceremonies but in public as a summer cap, especially in celebration of spring on the First of May [51]. Furthermore, Finnish students wear and extensively customise colourful student boiler suits [49]. This practice seems to have evolved out of the tradition of wearing boiler suits when visiting engineering facilities. Today student associations in Finland, Sweden and Canada have taken up these suits as unofficial uniforms and academic attire in student activities [49].

In Finnish higher education, guilds are associations composed of students of one faculty or study programme and provide various activities and facilities to their members throughout the academic year. These activities range from night-life, like pub-crawls and parties, to educational events, such as company visits or hackathons. These guilds hand out the student boiler suits (fi: opiskelijahaalarit) to their members (see figure 1), often referred to as "overalls". Each association's suit has a specific colour, the association's logo on the back and several sponsors distributed across. The primary function of the suit is as a display of affiliation, a uniform, somewhat comparable to ancient heraldry. Wearing a suit displays membership within student culture, and the colour signifies membership to a specific guild (see [35] or in Finnish [44] for more). This lively tradition of Finnish students offers potential space for exploring clothing practices because it has elements of a uniform but is intertwined with everyday public life.

\section{RESEARCH APPROACH}

As revealed through the current literature, design researchers have investigated how digital expression and other computational functions can become part of everyday life clothing. To study this in context, we selected a case of an existing culture around clothing practices. The unit of analysis is not a particular artefact, but what people do or imagine to do in practice [24]. We try to answer our research questions in two parts. In one part, we investigate current practices with ethnographic methods, mainly participant observation and interviews. In a second part, three dialogue-labs sessions generate speculative designs about future scenarios. Both methods combined help our reflection on designing social wearables. Figure 2 gives an overview of the data collection; however, we collected field notes throughout the timeline during smaller encounters. While results of the fieldwork informed the co-design sessions, the purpose goes beyond informing the design activities. To contextualise the findings of our co-design sessions, we will first report on our ethnographic fieldwork and findings and then the co-design procedure and outcomes. Finally, the discussion section of this paper connects both to formulate overarching themes.

\section{FIELD CASE STUDY: FINNISH UNIVERSITY STUDENT CULTURE}

In this chapter, we report the cultural insights we gained from conducting ethnographic fieldwork. The fieldwork extends the focus beyond wearables to allow the reader a better understanding of this particular culture of dress. These insights into existing practices of dress inform our co-design activities [3] and decipher the meanings, people's sentiments and ideas. As the primary author conducted the ethnographic fieldwork, this section is written in first person as a post-structural tale [47].

\subsection{Participants and Sites}

For ten months, I participated in various student activities (see figure 2), and through my workplace, at the university, I am confronted with the local student culture. As a graduate student, I joined the student association of my department (computer science) and acquired their boiler suit. I wore the suit in May Day celebrations and modified it during two sewing events - one of the clubs around handcrafts in computer science and one at the association of design students. Furthermore, I participated in a formal student banquet (en: sittning [50]) and interviewed people throughout these events and spontaneously on the universities campus. The fieldwork was recorded through secure digital notes, as typing on the phone seemed less intrusive than carrying a physical notebook. Audio memos before and after each field visit and photographs of garments and places supplemented these notes. After each field visit, I wrote down detailed field notes based on all material. Further, I interviewed four students, whose names are changed here, in-depth for their suit modifications and personal experiences inside the student culture. I chose to interview Teemu (bachelor student, computer science guild, 22) in a campus cafeteria, Matti (master 


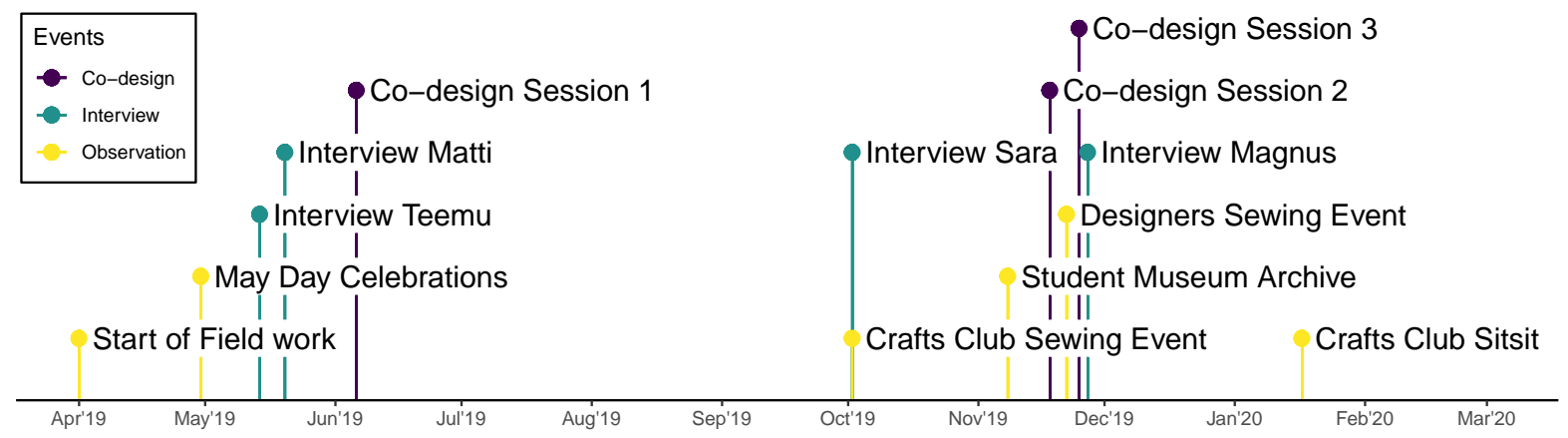

Figure 2: The timeline of highlights of data collection during 2019 and beginning of 2020 .

student, automation guild, 26) in his apartment, Magnus (bachelor student, bio-engineering, 26) at his workplace at the student union and Sara (automation and computer science guild, 23, female) during a sewing event. Those interviews were audio-recorded and transcribed. The collected data was analysed periodically for emerging themes, which then structured the ongoing data collection in shifting the focus of the observations and interviews. In contemporary ethnography, it is crucial to experience first-hand while staying aware of ones own personal influence [1,39]. Therefore I regularly reflected my experiences and findings with members of the research group in each analysis iteration. In this work, I will report only the themes of the fieldwork that describe the cultural practices around dress and identity towards our first research question.

\subsection{Practices around Boiler Suits and Dress in Finnish Student Culture}

4.2.1 Belonging and Brotherhood. As a migrant myself, seeing masses of students roaming around a city in colourful boiler suits might seem odd. Once I dressed up in my own overall and joined the students in their activities, it became apparent how this culture is similar to student culture in general. Wearing this overall is a statement of belonging to student culture and mediates a feeling of belonging the moment you slip into the overall's spacious garment. The overall functions as a very explicit symbol of membership. Although the colours of overalls differentiate guilds, wearing any overall makes you part of the community beyond your guild, your university, even your country.

Sara describes this "Teekkari spirit" (Teekkari is the Finnish term for university student of technology) as "community, hands-on and accepting people as they are". As a daughter of Chinese immigrants "being part of a community is important to [her]". She managed a student club providing baking and handicraft events and describes her motivation as "being able to contribute 'good vibes' to people". Similarly, Magnus describes the other members of his guild's board as "family".

One central practice with a long history in Nordic technology universities is the sittnings (fi: Sitsit). Those evening events revolve around a seated meal and drinking accompanied by toasts and singing in between servings. Those songs often reference the culture itself and praise themes of unity, brotherhood and drinking.

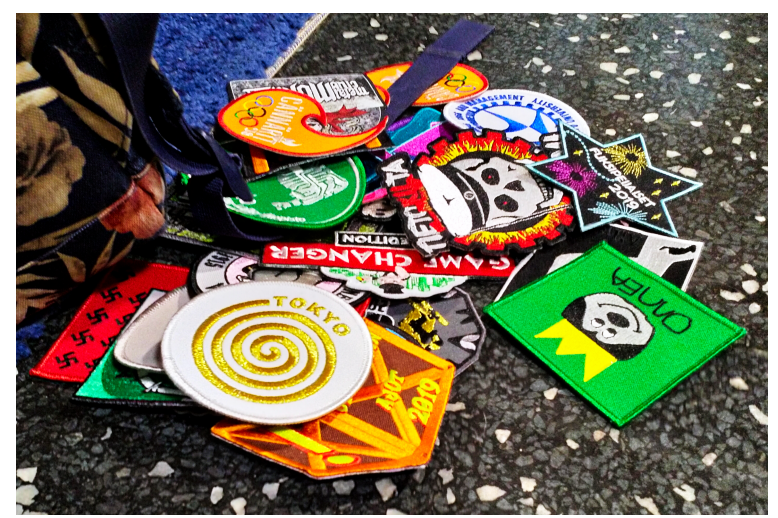

Figure 3: During a collective sewing event, a student brought a stack of cloth patches for sewing on their suits and trading with others.

When I participated in a sittning, I was surprised how the students welcomed me and exchange students in their ranks despite age or cultural differences.

4.2.2 Formalised Practices and Structures. Those sittnings are also an example of the formalised structures inherent to this culture. Each sittnings has at least one dedicated song master, who defines when people will sing and is responsible for the entertainment of the guests. Formal procedures specify, when to speak, when to sing and when to drink. The guilds also have formalised roles. In the first year, freshmen are called Fuksit (en: foxes), and one captain has the role of introducing them to the culture. A first-year student can collect points by attending guild activities to earn their right as a full member. For technology students, this ensures the right to wear a specific student cap starting at yearly festivities around May Day (fi: Vappu). This hierarchy is also visible in their suits. For example, a Fuksitcaptain wears a felt patch with a white " $F$ " (see Figure 1a). Similarly the students' outfits follow semi-formalised rules (see [35]), that define how to wear and modify their overalls.

4.2.3 Expressing Individuality. Despite conformity to the group many aspects also allow students to express their individuality. The 


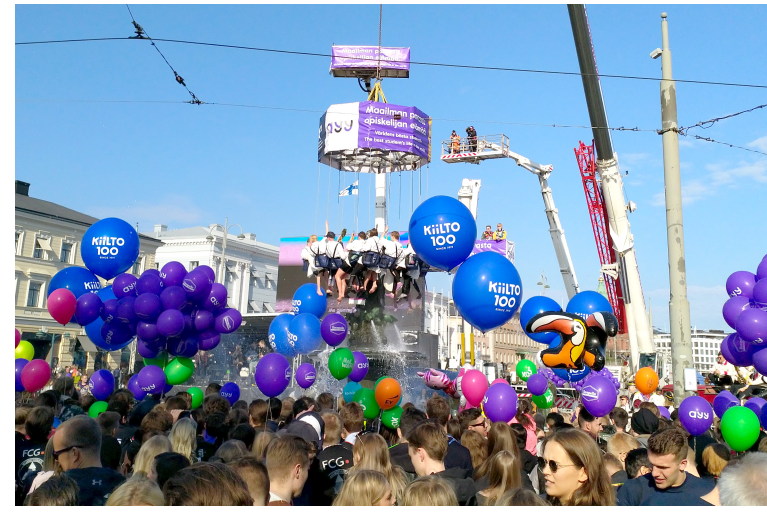

Figure 4: On the eve before the First of May students meet to celebrate the official start by gathering in the city centre and crowning a particular statue with a student cap.

overall functions as a social medium and a representation of individuality through the constant modifications. The suits are customised through patches and accessories by each student individually. One central modification seen on all suits is textile patches (shown in figure 3). Besides the student associations, many interest groups for hobbies, sports or culture have distinct logo patches. Other patches reveal participation in events or tours and in that way show a personal history. Many of these badges require competitions to earn them and therefore signify status within the culture, quite literally "badges of honour". Additionally, people attach patches portraying jokes or favourite characters from media. The practice is an ongoing process of finding new patches or trading them with "badge-dealers" or peers. Furthermore, students also modify the suit garments themselves. Exchanging a sleeve with their partner from a different coloured guild displays a relationship to that person (fig. 1b). Although some modifications are practical (e.g. adding an extra pocket), they are often personal and carefully crafted, like the hood with spikes in figure 1a. In recent years students have started integrating wearable technology in their suit, like Teemu's light-emitting belt in figure 1d. A student suit signifies membership to peer groups (e.g. joining an interest club), social status (e.g. freshmen captain) and individuality (e.g. light emitting belt). By curating the appearance of their suits, students create a representation of themselves. The suit's meaning is diverse and contextual and gives an identity inside this community.

4.2.4 Rowdiness and Testing Social Norms. Even though many practices remind of more formal organisational structures, a big part of the culture is humour. The start of May Day festivities, for example, starts with a "declaration of rowdiness". This rowdiness shows in performing pranks on each other, the lavish boiler suits and festive decorations (see figure 4). Further, some songs during sittings and many clothing patches play on dark humour. Magnus explained it as a way of using humour to overcome negative topics because they "become less serious when you joke about them". However, he decided against wearing a patch which plays on the infamous slogan of the German concentration camp Auschwitz, stating: "Grammatik macht frei” (en: Grammar sets you free). Such jokes might appear extreme to an outsider like me, but that is also its intended purpose. By touching controversial topics, students find and express their limits and consequently develop identity.

4.2.5 Creativity and Self. Finally, creativity plays a huge role in Teekkari and more general, Finnish university student culture. The students reinvent signs and symbols, thus understanding their meaning requires contextual knowledge. Some references are to specific aspects of the culture, as a tradition of one particular school or guild. Other contents reference internet and pop culture. For example, in figure $1 \mathrm{~d}$, the depiction of Donald Duck is copied from a Finnish webcomic on programming. Both Magnus and Matti have created and sold their patch designs. Moreover, Teemu and Matti already integrated electronics in their suits and they receive praise from the community for doing so. Teemu created his belt (fig. 1d) to "stand out" but also because he wanted to learn to build electronics himself. Magnus explained this tendency to create something as "leaving a mark". He described his feeling of pride in creating a new tradition in his guild. For the third year in a row, members of his guild went on a field trip he initiated.

4.2.6 Building Community and Identity between Tradition and Ingenuity. During my ethnography, I captured themes of sexual, vocational and group identity similar to my personal experience with student culture. The specific practices illustrate the more general practice of building identity. The students' actions play within the tension between formal structures and experimentation with new symbols and meaning. This duality corresponds with the duality of group belonging and individuality. With clothing, people follow known symbols to display belonging to a group, while at the same time differentiating from others. Further, the openness of the culture to novelty underlines the potential for innovation.

\section{CO-DESIGN SESSIONS}

This work is embedded in an ongoing research-through-design [42] project concerned with novel forms of social wearables based on the practices of dress of Finnish University students. The present study aims to imagine these novel forms based on the students' socio-cultural context. The dialogue-labs method [28] is an established approach to spark creativity and discussion in participation with users. We chose this co-design approach to include the students' perspective and generate insight into our research questions from the resulting discussion. The following section describes the different tasks and materials that facilitated the ideation in three co-design sessions.

\subsection{Participants}

We advertised the workshop with flyers, in person during our fieldwork and through mailing lists and social media channels across the university campus and student associations. Each co-design session was conducted by two designers of the research team and six, seven and eight student participants, respectively. The 21 participants $(6$ female) were students except for one postdoctoral HCI researcher (not part of the research group) aged from 19 to 32 years $(M=24)$. The students were on different levels (ten undergraduate, eight master, and two doctoral students) and had studied between one and nine years ( $M=3,5$ years). Participants mainly studied computer 
Table 1: The different co-design stations and which scenario and material they provided

\begin{tabular}{lll}
\hline Station & Scenario & Material / Method \\
\hline 1 & Alone on commute dressed in suit & Prototyping by cutting and colouring cotton \\
2 & Trip with close friend/partner & Sketching on canvas combining suit templates \\
3 & Enacting a prank with freshman team & Storyboard with PLEX cards \\
4 & Sittning: party with formal clothing and program & Brainstorming with PLEX cards \\
5 & First of May: yearly celebration of all students & Sketching on suit templates on a large wall \\
6 & Pub crawl: competitive events between freshman teams & Sketching with textile markers on a suit / textiles \\
\hline
\end{tabular}

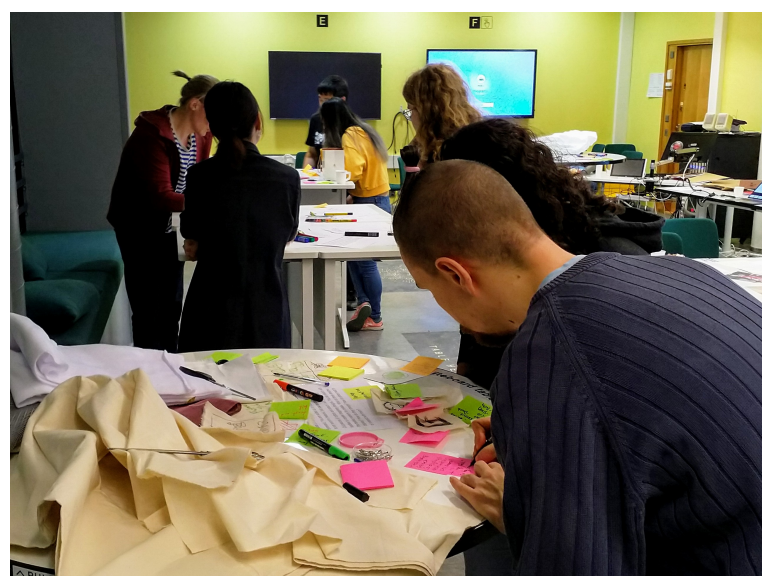

Figure 5: Co-design station 1 with the instructions, materials (cotton, scissors, textile markers, sticky notes and pens) and the ideas students created in the form of drawings and written notes.

science (12) but also electrical engineering (3), design (2), as well as one each automation science, bio-engineering, data science and mathematics. All but two participants had been active in student association activities, with 16 joining at least a few events each year. Fifteen people did customise their student outfits before, and eight participants had prior experience in designing wearable electronics in a university course or as a hobby (e.g. cosplay).

\subsection{Design Brief}

The main goal of the workshop dealt with imagining novel wearable technology that would be part of the students' dress and be used in face-to-face interactions. Therefore the main question presented to participants was: "How would you integrate wearable technology into your student outfits?" To sensitise the students for the topic we provided them with video material of state of the art technologies and the two design concepts "wearable expression" and "interactivity". The former introduced the questions: "If your suit could dynamically change its appearance, how would you want it to change? And what content do you want to share?" The latter introduced the question: "If your suits were interactive or connected to each other, how would you use them as a group?" These two potentials for design combine the fundamental aspects of social wearables [9].

\subsection{Scenarios and Materials}

According to the dialogue-labs method the room was prepared with six stations with different materials to work on the design brief. The stations introduced each a different scenario based on our ethnographic findings: a bar crawl, the First of May celebrations, a sittning, a prank, a trip with a close friend or partner and commuting alone. At each station a different material was provided, respectively: textile prototyping with cotton fabric and markers (see figure 10 as an example), sketching on a boiler suit, storyboard ideation with PLEX cards [26, 27], brainstorm with PLEX cards [26, 27], sketching on paper suit templates (see figure 9 as an example), sketching on a large canvas with paper templates. Table 1 lists all stations in detail. Additionally, some interactive wearable prototypes and smart textiles were spread across some of the stations. All stations included sticky notes and pens to document ideas as seen in Figure 5. These different materials and scenarios helped to generate a large variety of ideas. Many of the material properties are mere choices of convenience, not the essence of what is being prototyped. This similarly holds when the things being prototyped are services or practices [25].

\subsection{Procedure}

Each of the three workshops lasted two hours, organised into sections of 15 minutes. In the first quarter-hour, participants were welcomed, introduced to the study and asked to give written consent. Then one researcher introduced the design brief for the design activities, including the sensitising video material. After this first phase participants freely formed pairs for the co-design activity. Each pair performed three 15 minute co-design sessions on three of the six different stations of their own choice. The researchers facilitated the process by providing further context and reflection when needed. After these three iterations and a small break, the whole design group went through the six stations and the pairs presented their ideas over the course of 30 minutes. Here, the sharing of ideas seamlessly passed over into a group design process to develop the ideas further. In the last 15 minute phase, the whole group discussed the ideas for their relevance to the initial design brief and the participants themselves. Finally, the participants' reported their demographic data and evaluation of the ideas in a questionnaire. Participants chose the most promising idea from each station and rated it on a Likert scale from -3 to +3 .

\subsection{Analysis}

Similar to the dialogue-labs method [28], we identify the most valuable ideas based on participant ratings. Additionally the workshop 
was video and audio recorded to capture not just the designs but also the participant's qualitative notions towards those. After each session the two researchers discussed and noted initial themes. The idea sharing, group design and discussion phases were transcribed and analysed to reveal each idea and people's notion towards it. The mix of ideas between stations and different participants per session generated very similar or duplicate ideas. Therefore, three researchers grouped ideas into distinct design concepts.

\subsection{Social Wearable Design Concepts}

In this section we report the results generated in our three co-design sessions. Overall we recorded 81 individual ideas, of which 17 were rated $(M=1.69)$. Here, we describe the four design concepts with the highest-rated ideas and two additional concepts that reoccurred throughout all sessions.

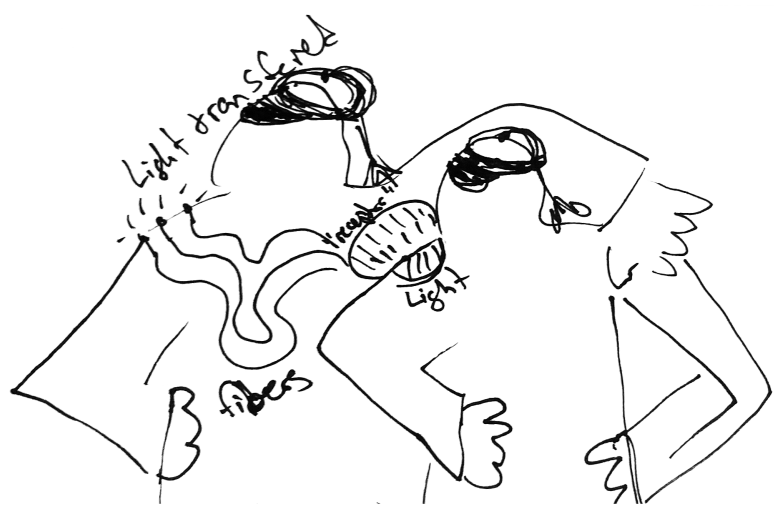

Figure 6: Sketch of the idea that overalls light up in a specific colour, when two people embrace each other

5.6.1 Displaying Groups Dynamically. One design concept, which encompasses a large number of ideas that came up in all three sessions, is to generate a shared display of visuals or sound by synchronising people in proximity. The principle behind those ideas is to create a shared experience by showing affiliation. For example, the overalls of a group standing together would light up in one colour to display their belonging to to each other (rated 2.83 in session 2(S2)). In figure 6 this is achieved through connecting overalls physically through a specific embrace. These visual synchronisations are also described to show visual animations or generative art on a massive crowd display. For example, a wearable display indicates blood alcohol levels during the yearly Vappu celebration in the central city square. Combined, the crowd generates one massive visualisation (rated 2.0 in S1). Further this can be used to synchronise musical activities. For example to harmonise the pitch while singing in a Sitsit (rated 2.125 in S1). The option to visualise groups based on proximity was thought as a possibility to differentiate groups, for example by each table in a Sitsit (rated 1.5 in S3). This could be used for competition and playfulness, e.g. loudest table in a sitsit lights the most.

5.6.2 Digital Badges as Collectables. The participants in all sessions seemed to appreciate ideas of interactive badges that enable the

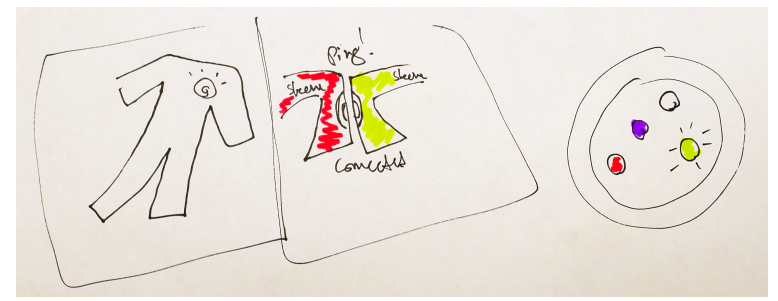

Figure 7: Sketch of a badge that adds the respective colour each time you bump sleeves with someone from another city or university.

exchange of content. One popular case was to use such digital badges for trading purposes. For example, a badge displays an entry ticket to an upcoming party and others then ask for that ticket (2.42 in S1). Participants also suggested collections or sets similar to trading card games. One idea included a competitive element, such that you can capture someone's badge. Participants highlighted the necessity of scarcity and temporality for making such digital content meaningful: "Five years ago Pokemon was something special, now everybody has it". Figure 7 shows one idea that of collecting for the purpose of diversification of a student's social networks. Here, a student can collect points by meeting and interacting with students from others groups, universities or cities. Further they described how the digital badge concept solves the problem of not having enough space for new patches left on the overall. This also sparked the ideas for dynamic advertisements, as the sponsors' logos already take up a lot of space in the students' overalls. However, some participants refused the idea of selling ad-space out of the concern of commercialisation.

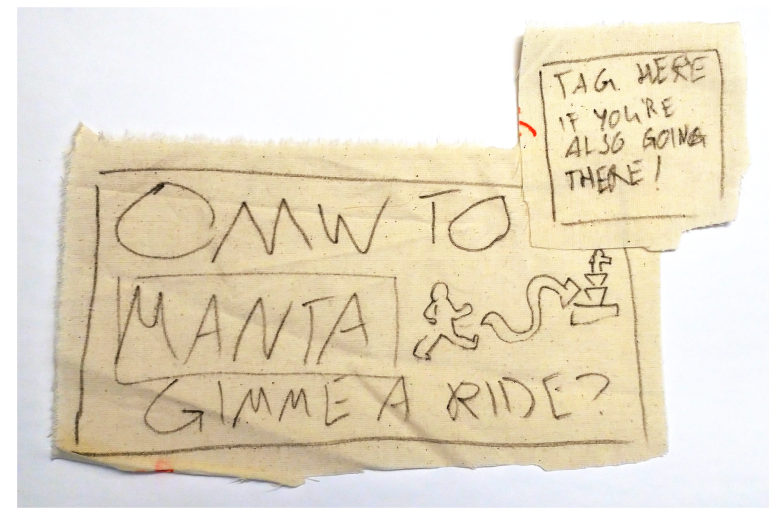

Figure 8: Sketch on textile that shows the currently planned activity and a function to join by tagging.

5.6.3 Personal Displays as Conversation Starters. One concept shown in many ideas of the participants was about displaying information as a conversation starter. Several ideas revolved around displaying personal information or conversation topics. Other ideas showed activity data, like blood alcohol levels or how many people someone 
met. One highly appreciated idea was to display your currently planned activities, such that others might join (rated 2.17 in S3, see fig. 8). Some ideas went even further and suggested to signal willingness to talk in form of a "traffic light" that displays mood (rated 2.16 in S2). This way people could manage their social interactions. For example, on the way home after partying strangers would know not to start a conversation.

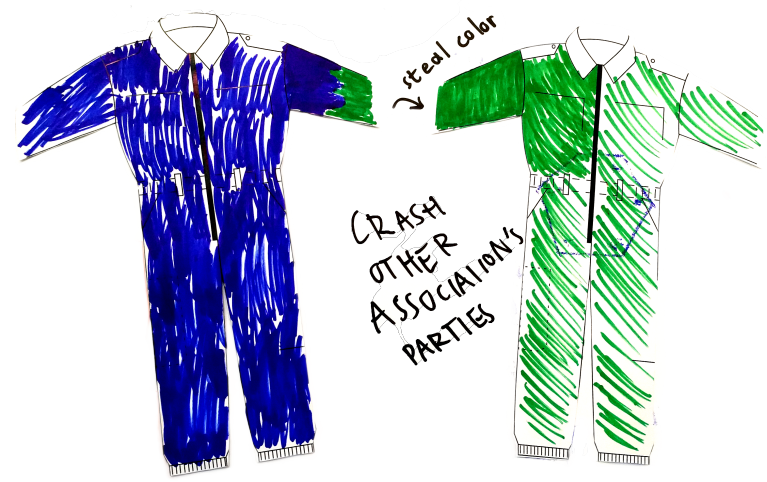

Figure 9: "Infiltrator overall" idea created at station 5 using the suit templates: Stealing the colour of another guild to sneak into private events.

5.6.4 Concealing Identity - Camouflage. In the first two sessions different participants generated the same concept of concealing your affiliation by changing the colour of your overall. As guilds identify by their colour, an idea was to appropriate the colour of another guild to infiltrate exclusive parties (see figure 9) (rated 2 in s1). They also imagined to blame other guilds for their own wrong-doings. One participant explained how their guild uses leftover suits from their former guild to dress up "when we're doing something stupid." Accordingly, a dynamic suit enables "adaptive blaming, so you can change it on the go". However, the same participant refused these "infiltration" suits, because "the colours lose their meaning." The meaning derives from the the colour being used as a symbol for belonging to one's guild. Making the colour dynamic consequently defeats that purpose.

5.6.5 Drawing Attention. A concept that repeated throughout all three sessions was about drawing attention. Most ideas of this kind incorporated light effects to highlight someone, for example through flashing a digital screen or light-emitting diodes in a group's logo. More so, people thought of tangible modalities, like fireworks or the tail in fig. 10, which shoots confetti, when pulled. This was seen as a way to guide group interactions. For example, a person could be highlighted to start singing a song. One participant expressed their dislike over the idea of using music to draw attention, as this would infringe her personal space too much.

5.6.6 Crowd-sourced safety. The aspect of personal safety repeated across all sessions and appeared in eight design ideas. Participants wanted to be safe from unwanted social interactions, like uncomfortable conversations, groping (see fig. 11) or bodily harm, and

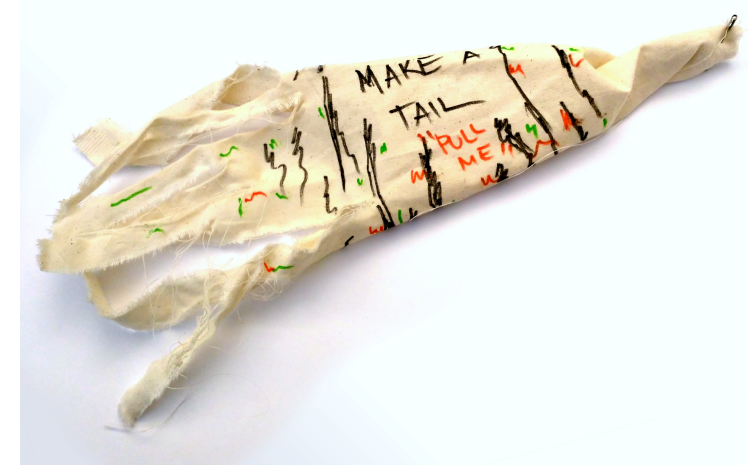

Figure 10: A textile prototype of a tail attachment that shoots confetti when pulled.

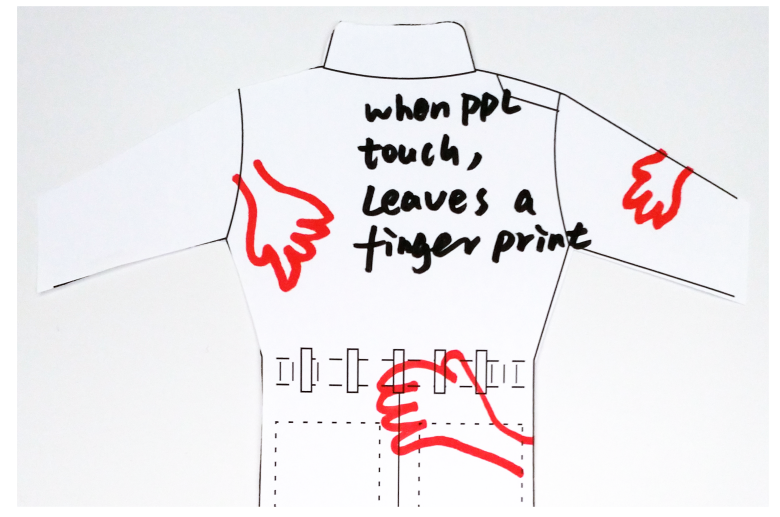

Figure 11: Sketch of an overall that leaves colourful marks, when someone is touched indecently.

avoid injuries due to drinking or other endangering activities. To prevent such incidents, participants generated ideas of people supporting each other through wearables. Such crowd-sourced safety included tracking people for any incidents and reporting those events to others through the overalls.

\section{DISCUSSION}

In this section, we reflect on the design concepts students generated, as well as on what we learned about the practices in Finnish student culture. All results together present new potential and challenges for the design of social wearables.

\subsection{Themes of Self-expression and Developing Identity}

Throughout our study, we discovered the themes of standing out and fitting in. Both practices are ways of shaping identity. By using symbols of a group, people demonstrate belonging to that group. At the same time, they differentiate themselves from others by making such statements about affiliation [5]. We will present three distinct characteristics of social wearables that contribute to these practices. 
6.1.1 Standing out through Versatility. The most obvious way of standing out is represented by the students' designs to draw attention. Plenty of existing designs in research and fashion have used this opportunity of spectacle, novelty and drawing attention to oneself [9]. These designs explored technical feasibility or placement in a context of performing arts. Our study adds a case where this applies in the life of university students. Another opportunity of social wearables for standing out is the extended versatility of digital expression over static garments. In the design concept of computational information for ice-breaking (see section 5.6.3) students used social wearables to adapt to context dynamically Similarly, in the concept of digital clothing patches (see section 5.6.2) students used them to overcome the limited space on their overalls. While prior work had revealed problems with digital aesthetics in clothing [10], our work did not show such reservations. The particular community around wearing overalls favoured a perception of being "techy". Therefore, we provide a particular case for designing for "spectacle" [9] with digital aesthetics.

6.1.2 Dynamically Display Belonging. The design concept of the group displays (see section 5.6.1) gives an explicit example of a social practice enhanced through wearable technology. Showing group belonging is a central aspect of clothing and fashion, and social wearables could intensify symbols by emitting light, sound or other modalities. The ideas that students described address this as an embodied feeling of togetherness. Furthermore, the design concepts and social practices showed various competitive elements. This competitiveness showed in rivalry with other groups through the group displays (section 5.6.1), outdoing other guilds through a digital disguise (section 5.6.4) or surpassing other students through collecting badges, analogue or digital. Each of these examples enable competition and consequently, provide means for distinction.

6.1.3 Meaning-Making in Digital Expression. The students also valued the versatility of digital expression because standing out requires them to display topical symbols. However, in the two design concepts Digital Badges as Collectables (section 5.6.2) and Concealing Identity - Camouflage (section 5.6.4) students exposed the problem of abundance. The symbolic meaning people associate with clothing patches stems from their rarity and context. Being able to copy any content between their outfits would remove those characteristics and reduce their meaningfulness. One solution could be Baytaş and colleagues' concept of digital heirlooms by limiting digital "material" through blockchain technology [2]. Limiting the circulation of digital content through crowd-based authorisation would reflect how clothing badges become meaningful. Our work delivers a suitable case for collecting and trading of digital content for self-expression with wearables.

\subsection{Towards Digital Expression with Social Wearables}

Based on our findings, we understand the student boiler suits as a social medium. Each suit represents the social networks of the wearer and enables self-expression through personalisation. The themes we revealed from our co-design results show how social wearables would extend people's self-expression practices. Prior work has argued that wearables need to integrate their technical function with the social functions of dress [45]. Fashion as a social function of dress is about the "symbolic belonging to a group to enable and execute self-expression as a member of a desired social group." $[4,43]$ Our study attributes this function to social wearables. Therefore, we see wearables for collocated social interactions as a way to replace or reinforce the social functions of dress.

Our case shows symbolic meaning is contextual and intrinsic to social interactions with wearables. This suggests that future designs have to allow people to reconfigure functionality. Dagan et al. hint towards this with their guiding question: "Are the sensing and/or actuation methods customizable by the wearer?" Our empirical findings highlight this as a criterion for wearables that aim for self-expression. Designing such social wearables should allow people to create meaning through their practices and avoid predefined labels (e.g. gender) or predefined interactions (e.g. groping $v s$. group touch). Social wearables have to negotiate between extending forms of expression and without limiting meaningful and respectful practices.

\subsection{Ethical Considerations on Practices with Social Wearables}

We also want to raise ethical concerns faced in our research. Although the field observations did not reveal gender issues in-situ, some ideas from the workshops revealed a conflict between gender roles, e.g. groping. The culture we investigated is historically maledominant and shows elitist structures. However, Finland ranks as one of the countries with the highest gender equality and problems like male-only student clubs are denounced publicly. Nevertheless, when we consider social wearables as a tool for people to shape their identities, designers need to be sensitive to existing cultural inequalities. It is plausible to anticipate that new social wearables might reinforce harmful practices, like discrimination and peerpressure. Other examples are the ideas which protect people when they are drunk. Interestingly Participant 2 interpreted a fall detection system as "You can drink safely because someone is going to find you". Consequently, we call for design to reflect on the practices that people do and actively introduce higher goals than technological advancement, such as sustainability [25] and freedom of expression [43].

\subsection{Future Work}

In our ongoing research we develop designs into working prototypes together with students. This ensures a high chance to create artefacts that are meaningful to participants. These prototypes will allow evaluating new practices with social wearables placed in the everyday lives of Finnish students and show us how practices with social wearables develop. Additionally, culture forms an integral part of our study. Therefore, it would be interesting to look at similar design concepts within a different social context of dress.

\section{CONCLUSION}

Through an ethnographic fieldwork and co-design activities, our paper introduces a case of practice-oriented design for social wearables. Here we look into the specific social context of Finnish students and how boiler suits can be modified using wearable computing technology. We observed existing practices of social identity 
in this student culture and technology use in clothing. Through co-design activities based on dialogue-labs, we generated six valuable design concepts. Drawing from those findings, we found that wearables offer versatility to stand out as an individual and display belonging to a social group dynamically. However, mediating between standing out and belonging should be facilitated by the design. We extend the argument that digital aesthetics could become part of everyday clothing. Reflecting upon this, we emphasise the value of designing for specific subcultures and argue that social wearables need to allow for reconfiguration of their functioning.

It is in this way, wearable designs should be able to not only juxtapose technological and social functions but perform the same social functions as clothing. As technologies become further integrated into our personal items, glasses and clothes we wear, the implications of wearable designs and practices have far-reaching social consequences.

\section{ACKNOWLEDGMENTS}

We want to thank the various individuals involved in the student association activities for their support in revealing this interesting case and sharing their personal perspectives. This work was supported by the Academy of Finland project Digital Aura [311090].

\section{REFERENCES}

[1] Michael Angrosino. 2007. Doing Ethnographic and Observational Research. SAGE Publications Ltd, 1 Oliver's Yard, 55 City Road, London EC1Y 1SP United Kingdom. https://doi.org/10.4135/9781849208932

[2] Mehmet Aydin Baytaş, Aykut Coşkun, Asim Evren Yantaç, and Morten Fjeld. 2018 Towards Materials for Computational Heirlooms: Blockchains and Wristwatches. In Proceedings of the 2018 Designing Interactive Systems Conference (DIS '18). Association for Computing Machinery, Hong Kong, China, 703-717. https: //doi.org/10.1145/3196709.3196778

[3] Jeanette Blomberg and Helena Karasti. 2012. Ethnography. In Routledge International Handbook of Participatory Design. Routledge. https://doi.org/10.4324/ 9780203108543.ch5

[4] Pierre Bourdieu. 1977. Outline of a Theory of Practice (25. printing ed.). Number 16 in Cambridge Studies in Social and Cultural Anthropology. Cambridge Univ. Press, Cambridge.

[5] Pierre Bourdieu and Richard Nice. 1984. Distinction: A Social Critique of the fudgement of Taste (reprint 2008 ed.). Routledge, New York.

[6] Jay Chen and Azza Abouzied. 2016. One LED Is Enough: Catalyzing Face-to-Face Interactions at Conferences with a Gentle Nudge. In Proceedings of the 19th ACM Conference on Computer-Supported Cooperative Work \& Social Computing - CSCW '16. ACM Press, San Francisco, California, USA, 172-182. https://doi.org/10.1145/ 2818048.2819969

[7] Ashley Colley, Minna Pakanen, Saara Koskinen, Kirsi Mikkonen, and Jonna Häkkilä. 2016. Smart Handbag as a Wearable Public Display - Exploring Concepts and User Perceptions. In Proceedings of the 7th Augmented Human International Conference 2016 on - AH '16. ACM Press, Geneva, Switzerland, 1-8. https //doi.org/10.1145/2875194.2875212

[8] Ella Dagan, Elena Márquez Segura, Ferran Altarriba Bertran, Miguel Flores, and Katherine Isbister. 2019. Designing 'True Colors': A Social Wearable That Affords Vulnerability. In Proceedings of the 2019 CHI Conference on Human Factors in Computing Systems (CHI '19). ACM, New York, NY, USA, 33:1-33:14. https //doi.org/10.1145/3290605.3300263

[9] Ella Dagan, Elena Márquez Segura, Ferran Altarriba Bertran, Miguel Flores, Robb Mitchell, and Katherine Isbister. 2019. Design Framework for Social Wearables. In Proceedings of the 2019 on Designing Interactive Systems Conference (DIS '19). ACM, New York, NY, USA, 1001-1015. https://doi.org/10.1145/3322276.3322291

[10] Laura Devendorf, Kimiko Ryokai, Joanne Lo, Noura Howell, Jung Lin Lee, Nan Wei Gong, M. Emre Karagozler, Shiho Fukuhara, Ivan Poupyrev, and Eric Paulos. 2016. "I Don't Want to Wear a Screen": Probing Perceptions of and Possibilities for Dynamic Displays on Clothing. In Proceedings of the 2016 CHI Conference on Human Factors in Computing Systems - CHI '16 (CHI '16). ACM Press, New York, NY, USA, 6028-6039. https://doi.org/10.1145/2858036.2858192

[11] Christine Dierk, Molly Jane Pearce Nicholas, and Eric Paulos. 2018. AlterWear Battery-Free Wearable Displays for Opportunistic Interactions. In Proceedings of the 2018 CHI Conference on Human Factors in Computing Systems - CHI '18. 1-11. https://doi.org/10.1145/3173574.3173794

[12] Lucy Dunne, Halley Profita, and Clint Zeagler. 2014. Social Aspects of Wearability and Interaction. In Wearable Sensors. Elsevier, 25-43. https://doi.org/10.1016/ B978-0-12-418662-0.00026-X

[13] Joanne Entwistle. 2015. The Fashioned Body: Fashion, Dress and Modern Social Theory (2. ed ed.). Polity Press, Cambridge.

[14] Sue Fairburn, Josie Steed, and Janet Coulter. 2016. Spheres of Practice for the Co-Design of Wearables. Fournal of Textile Design Research and Practice 4, 1 (Jan. 2016), 85-109. https://doi.org/10.1080/20511787.2016.1255445

[15] Jennica Falk and Staffan Björk. 1999. The BubbleBadge: A Wearable Public Display. CHI'99 extended abstracts on Human factors in computing systems (1999), 318-319. https://doi.org/10.1145/632716.632909

[16] Joseph Ferenbok, Steve Mann, and Katina Michael. 2016. The Changing Ethics of Mediated Looking: Wearables, Veillances, and Power. IEEE Consumer Electron. Mag. 5, 2 (April 2016), 94-102. https://doi.org/10.1109/MCE.2016.2516139

[17] Anna Fuste and Chris Schmandt. 2018. ARTextiles for Promoting Social Interactions Around Personal Interests. In Extended Abstracts of the 2018 CHI Conference on Human Factors in Computing Systems - CHI '18. ACM Press, Montreal QC, Canada, 1-6. https://doi.org/10.1145/3170427.3188589

[18] Çağlar Genç, Oğuz Turan Buruk, Sejda İnal Yılmaz, Kemal Can, and Oğuzhan Özcan. 2018. Recommendations for Designing Fashionable Wearables. International Journal of Design 12, 3 (2018), 1-19.

[19] Erving Goffman. 1959. The Presentation of Self in Everyday Life (nachdr. ed.). Doubleday, New York, NY.

[20] Emmi Harjuniemi, Ashley Colley, Piia Rytilahti, Hong Li, Jesse Forest, and Jonna Häkkilä. 2018. Idle Stripes Shirt: Ambient Wearable Display for Activity Tracking. In Proceedings of the 2018 ACM International Symposium on Wearable Computers (ISWC '18). ACM, New York, NY, USA, 254-259. https://doi.org/10.1145/3267242. 3267303

[21] Katherine Isbister, Kaho Abe, and Michael Karlesky. 2017. Interdependent Wearables (for Play): A Strong Concept for Design. In Proceedings of the 2017 CHI Conference on Human Factors in Computing Systems (CHI '17). ACM, New York, NY, USA, 465-471. https://doi.org/10.1145/3025453.3025939

[22] Pradthana Jarusriboonchai, Felix Anand Epp, Thomas Olsson, Andrés Lucero, Oscar Tomico, Eric Paulos, and Jonna Häkkilä. 2019. Beyond Individuals: Exploring Social Experiences Around Wearables. In UbiComp/ISWC '19 Adjunct (UbiComp/ISWC '19). ACM, New York, NY, USA, 454-456. https://doi.org/10. $1145 / 3341162.3347754$

[23] Hsin-Liu (Cindy) Kao, Deborah Ajilo, Oksana Anilionyte, Artem Dementyev, Inrak Choi, Sean Follmer, and Chris Schmandt. 2017. Exploring Interactions and Perceptions of Kinetic Wearables. In Proceedings of the 2017 Conference on Designing Interactive Systems - DIS '17. ACM Press, Edinburgh, United Kingdom, 391-396. https://doi.org/10.1145/3064663.3064686

[24] Kari Kuutti and Liam J. Bannon. 2014. The Turn to Practice in HCI: Towards a Research Agenda. In Proceedings of the SIGCHI Conference on Human Factors in Computing Systems (CHI '14). ACM, New York, NY, USA, 3543-3552. https: //doi.org/10.1145/2556288.2557111

[25] Lenneke Kuijer. 2017. Practices-Oriented Design. In Design for Behaviour Change. Taylor and Francis Ltd., United Kingdom, 116-127. https://doi.org/10.4324/ 9781315576602

[26] Andrés Lucero. 2019. PLEX Cards · Playful Experiences Cards. Retrieved 2019-09-24 from http://www.funkydesignspaces.com/plex/

[27] Andrés Lucero and Juha Arrasvuori. 2010. PLEX Cards: A Source of Inspiration When Designing for Playfulness. In Proceedings of the 3rd International Conference on Fun and Games (Fun and Games '10). ACM, New York, NY, USA, 28-37. https: //doi.org/10.1145/1823818.1823821

[28] Andrés Lucero, Kirsikka Vaajakallio, and Peter Dalsgaard. 2012. The DialogueLabs Method: Process, Space and Materials as Structuring Elements to Spark Dialogue in Co-Design Events. CoDesign 8, 1 (March 2012), 1-23. https://doi. org/10.1080/15710882.2011.609888

[29] Angella Mackey, Ron Wakkary, Stephan Wensveen, and Oscar Tomico. 2017. "Can I Wear This?" Blending Clothing and Digital Expression by Wearing Dynamic Fabric. International fournal of Design 11, 3 (2017), 15. http://www.ijdesign.org/ index.php/IJDesign/article/view/3177/794

[30] Elena Márquez Segura, James Fey, Ella Dagan, Samvid Niravbhai Jhaveri, Jared Pettitt, Miguel Flores, and Katherine Isbister. 2018. Designing Future Social Wearables with Live Action Role Play (Larp) Designers. In Proceedings of the 2018 CHI Conference on Human Factors in Computing Systems (CHI '18). ACM, New York, NY, USA, 462:1-462:14. https://doi.org/10.1145/3173574.3174036

[31] Matthew Mauriello, Michael Gubbels, and Jon E. Froehlich. 2014. Social Fabric Fitness: The Design and Evaluation of Wearable E-Textile Displays to Support Group Running. In Proceedings of the 32nd Annual ACM Conference on Human Factors in Computing Systems - CHI '14. ACM Press, Toronto, Ontario, Canada, 2833-2842. https://doi.org/10.1145/2556288.2557299

[32] Donald McMillan, Barry Brown, Airi Lampinen, Moira McGregor, Eve Hoggan, and Stefania Pizza. 2017. Situating Wearables: Smartwatch Use in Context. In CHI '17. ACM Press, 3582-3594. https://doi.org/10.1145/3025453.3025993 
[33] Trine Møller. 2018. Presenting The Accessory Approach: A Start-up's Journey Towards Designing An Engaging Fall Detection Device. In Proceedings of the 2018 CHI Conference on Human Factors in Computing Systems (CHI '18). ACM, New York, NY, USA, 559:1-559:10. https://doi.org/10.1145/3173574.3174133

[34] Vivian Genaro Motti and Kelly Caine. 2015. Users' Privacy Concerns About Wearables. In Financial Cryptography and Data Security (Lecture Notes in Computer Science), Michael Brenner, Nicolas Christin, Benjamin Johnson, and Kurt Rohloff (Eds.). Springer Berlin Heidelberg, 231-244.

[35] Nelli Lapintie. 2018. Student Overalls: 10 Essential Rules. https://www.tylkkari. fi/english/student-overalls-10-essential-rules

[36] Thomas Olsson, Pradthana Jarusriboonchai, Paweł Woźniak, Susanna Paasovaara, Kaisa Väänänen, and Andrés Lucero. 2019. Technologies for Enhancing Collocated Social Interaction: Review of Design Solutions and Approaches. Comput Supported Coop Work (Feb. 2019). https://doi.org/10.1007/s10606-019-09345-0

[37] Eric Paulos and Elizabeth Goodman. 2004. The Familiar Stranger: Anxiety, Comfort, and Play in Public Places. In Proceedings of the SIGCHI Conference on Human Factors in Computing Systems (CHI '04). ACM, New York, NY, USA 223-230. https://doi.org/10.1145/985692.985721

[38] Jennifer Pearson, Simon Robinson, and Matt Jones. 2015. It's About Time Smartwatches as Public Displays. In CHI '15. ACM Press, 1257-1266. https: //doi.org/10.1145/2702123.2702247

[39] Sarah Pink. 2009. Doing Sensory Ethnography. Sage Publications, London ; Thousand Oaks, CA.

[40] Inka Rantala, Ashley Colley, and Jonna Häkkilä. 2018. Smart Jewelry: Augmenting Traditional Wearable Self-Expression Displays. In Proceedings of the 7th ACM International Symposium on Pervasive Displays - PerDis '18. ACM Press, Munich, Germany, 1-8. https://doi.org/10.1145/3205873.3205891

[41] Lise Skov and Marie Riegels Melchior. 2010. Research Approaches to the Study of Dress and Fashion. Berg Encyclopedia of World Dress and Fashion: Global Perspectives 10 (2010), 11-16. https://research.cbs.dk/en/publications/researchapproaches-to-the-study-of-dress-and-fashion

[42] Pieter Jan Stappers and Elisa Giaccardi. 2017. Research through Design. In The Encyclopedia of Human-Computer Interaction. https://www.interactiondesign.org/literature/book/the-encyclopedia-of-human-computer-interaction2nd-ed/research-through-design

[43] Sakari Tamminen and Elisabet Holmgren. 2016. The Anthropology of Wearables The Self, The Social, and the Autobiographical. Ethnographic Praxis in Industry Conference Proceedings 2016, 1 (Nov. 2016), 154-174. https://doi.org/10.1111/1559-
8918.2016.01083

[44] Tekniikan akateemiset TEK. 2017. Teekkarit. Retrieved 2020-01-30 from https://www.teekkarit.fi/

[45] Oscar Tomico, Lars Hallnäs, Rung-Huei Liang, and Stephan A G Wensveen. 2017. Towards a Next Wave of Wearable and Fashionable Interactions. IfDesign 11, 3 (2017), 6 .

[46] Muhammad Umair, Muhammad Hamza Latif, and Corina Sas. 2018. Dynamic Displays at Wrist for Real Time Visualization of Affective Data. In Proceedings of the 19th International ACM SIGACCESS Conference on Computers and Accessibility - DIS '18. ACM Press, Hong Kong, China, 201-205. https://doi.org/10.1145/ 3197391.3205436

[47] John Van Maanen. 2011. Tales of the Field: On Writing Ethnography (2nd ed ed.). University of Chicago Press, Chicago.

[48] Yanan Wang, Shijian Luo, Yujia Lu, Hebo Gong, Yexing Zhou, Shuai Liu, and Preben Hansen. 2017. AnimSkin: Fabricating Epidermis with Interactive, Functional and Aesthetic Color Animation. In Proceedings of the 2017 Conference on Designing Interactive Systems (DIS '17). ACM, New York, NY, USA, 397-401. https://doi.org/10.1145/3064663.3064687

[49] Wikipedia. 2019. Student Boilersuit. Wikipedia (Sept. 2019). https://en.wikipedia. org/w/index.php?title=Student_boilersuit\&oldid=917704711

[50] Wikipedia. 2020. Sittning. Wikipedia (May 2020). https://en.wikipedia.org/w/ index.php?title=Sittning\&oldid $=957320534$

[51] Wikipedia. 2020. Student Cap. Wikipedia (Jan. 2020). https://en.wikipedia.org/ w/index.php?title=Student_cap\&oldid=937479371

[52] Wikipedia. 2020. Varsity Letter. Wikipedia (May 2020). https://en.wikipedia.org/ w/index.php?title=Varsity letter\&oldid $=955325723$

[53] Volker Wulf, Claudia Müller, Volkmar Pipek, David Randall, Markus Rohde, and Gunnar Stevens. 2015. Practice-Based Computing: Empirically Grounded Conceptualizations Derived from Design Case Studies. In Designing Socially Embedded Technologies in the Real-World, Volker Wulf, Kjeld Schmidt, and David Randall (Eds.). Springer London, London, 111-150. https://doi.org/10.1007/9781-4471-6720-4_7

[54] Clint Zeagler. 2017. Where to Wear It: Functional, Technical, and Social Considerations in on-Body Location for Wearable Technology 20 Years of Designing for Wearability. In Proceedings of the 2017 ACM International Symposium on Wearable Computers - ISWC '17. ACM Press, Maui, Hawaii, 150-157. https://doi.org/10.1145/3123021.3123042 